\title{
Adsorption of $\mathrm{H}_{2}, \mathrm{O}_{2}, \mathrm{H}_{2} \mathrm{O}, \mathrm{OH}$ and $\mathrm{H}$ on monolayer $\mathrm{MoS}_{2}$
}

\author{
F Ferreira ${ }^{1}$, A Carvalho ${ }^{2}$, Í J M Moura ${ }^{3}, \mathrm{~J} \mathrm{Coutinho}^{4}$ and R M \\ Ribeiro $^{1}$ \\ ${ }^{1}$ Centro de Física and Departamento de Física and QuantaLab, Universidade do \\ Minho, Campus de Gualtar, Braga 4710-057, Portugal \\ ${ }^{2}$ Centre for Advanced 2D Materials and Graphene Research Centre, National \\ University of Singapore, Singapore 117542, Singapore \\ E-mail: ricardo@fisica.uminho.pt \\ ${ }^{3}$ Centro de Física and Departamento de Física and QuantaLab, Universidade do \\ Minho, Campus de Gualtar, Braga 4710-057, Portugal; CAPES Foundation, Ministry \\ of Education of Brazil, Brasília - DF, 70040-020, Brazil. \\ ${ }^{4}$ Department of Physics and I3N, University of Aveiro, Campus Santiago, 3810-193 \\ Aveiro, Portugal.
}

\begin{abstract}
Hydrogen and hydrogen-containing gases are commonly used as reductants in chemical vapor deposition (CVD) growth of $\mathrm{MoS}_{2}$. Here, we consider the defects resulting from the presence of hydrogen during growth and the resulting electronically active defects. In particular, we find that the interstitial hydrogen defect is a negative- $U$ center with amphoteric donor and acceptor properties. Additionally, we consider the effects of the interaction with water and oxygen. The defects are analysed using density functional theory calculations.
\end{abstract}

PACS numbers: $68.43 . \mathrm{Bc}, 68.43 . \mathrm{Fg}, 74.20 . \mathrm{Pq}$

$\mathrm{MoS}_{2}$, Adsorption, DFT 


\section{Introduction}

The transition metal dichalcogenide $\mathrm{MoS}_{2}$ is amongst the most studied two-dimensional semiconductors, due to its optical bandgap in the visible range $(1.8 \mathrm{eV})$, and the possibility of selective optical excitation of spin and valley states with potential applications to information processing.

In recent years, efforts have been made to develop cheap and scalable methods to grow $2 \mathrm{D}$ materials having in view the possibility of industrial device production. The chemical vapor deposition (CVD) approach is one of the most promising for the production of graphene, $\mathrm{MoS}_{2}$ and other chalcogenides, boron nitride, amongst others. Recently, several groups have produced $\mathrm{MoS}_{2}$ mono or few layers using CVD.[Lee and et al.(2012), Kim and et al.(2015), Kim and et al.(2016), Mohapatra and et al.(2016)] The operation parameters of the method, including percursors, growth atmosphere, etc., vary, and are critical for the crystallinity and purity of the material grown. $\mathrm{MoS}_{2}$ is typically produced by reduction of $\mathrm{MoO}_{3}$ or Mo by sulfurization with sulfur or $\mathrm{H}_{2} \mathrm{~S}$.[Kim and et al.(2016)] Different from the growth of graphene, the addition of $\mathrm{H}_{2}$ to the $\mathrm{MoS}_{2}$ growth atmosphere was found to inhibit the thermal-induced etching process during film growth and promote desulfurization and, in large concentrations, to damage the continuity of the film.[Li(2015)] Appart from direct addition of $\mathrm{H}_{2}$ or $\mathrm{H}_{2} \mathrm{~S}$, hydrogen can also be inadvertedly incorporated during the various processing steps to which the film is submited from growth to device fabrication.

Atmospheric oxygen is normaly believed to heal vacancies and other defects resulting from sulphur deficiency in $\mathrm{MoS}_{2}$. $[\mathrm{Ma}(2016)]$ Oxygen-assisted CVD growth has also been reported to result in improved domain growth and crystallinity.[Chen(2015)] However, if the material is exposed to oxygen plasma together with hydrogen treatment, reactive sites are introduced instead.[Ye(2016)] However, little is known about the role of oxygen and hydrogen defects in $\mathrm{CVD} \mathrm{MoS}_{2}$, as these small elements escape detection in atomically resolved scanning electron microscopy or scanning tunneling microscopy. Clearly, however, the structure of the defects involved and the respective reactions need to be better described in order for the reactivity and electronic character of the resulting $\mathrm{MoS}_{2}$ material to be controllable, to produce either chemically active substrates for catalysis applications or highly crystalline, passivated semiconductors for electronic and optoelectronic applications.

In this work, we do non colinear density functional theory with spin-orbit calculations to study the adsorption of $\mathrm{H}_{2}, \mathrm{O}_{2}, \mathrm{OH}, \mathrm{H}_{2} \mathrm{O}$ molecules and atomic $\mathrm{H}$ on the $\mathrm{MoS}_{2}$ monolayer. The spin-orbit coupling is needed to obtain the correct electronic structure, and has little effect in the interaction between the substrate and the adsorbants. In Section 2 we describe the method. In Section 3 we present the results and discuss them, starting by the configurations the molecules adopt on the surface and its energetics. Then we look at the resulting electronic structures. 


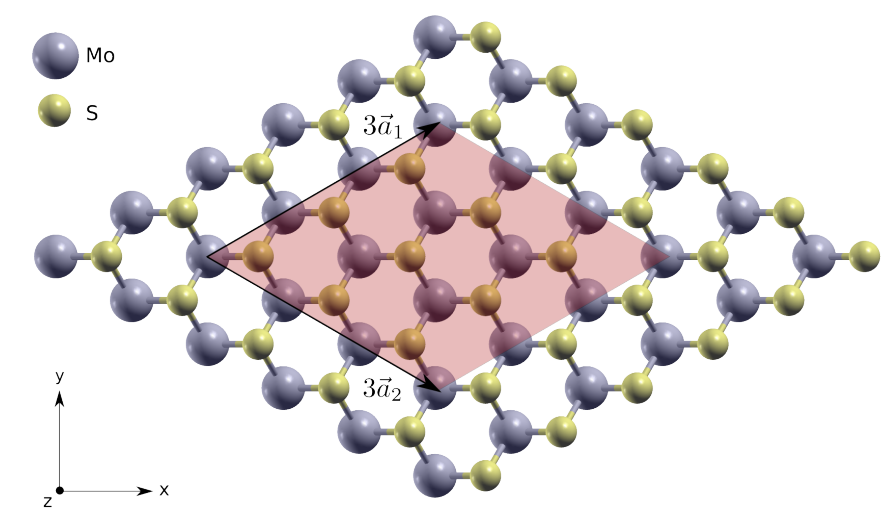

Figure 1. (Color online) Monolayer $\mathrm{MoS}_{2}$. The red shadow region is the area of the supercell considered; $3 \vec{a}_{1}$ and $3 \vec{a}_{2}$ are the lattice vectors of the supercell.

\section{Method}

Calculations were performed using non collinear density-functional theory (DFT) with spin-orbit interaction with the open source software QUANTUM ESPRESSO.[Giannozzi and et al.(2009)] The exchange-correlation functional used was the generalized gradient approximation of Perdew-Burke-Ernzerhof (GGA-PBE).[Perdew and et al.(1996)] We included a van der Waals interaction through the semi-empiric dispersion term of Grimme.[Grimme(2006), Barone and et al.(2009)] A cut-off energy of 50 Ry is used for all configurations, except for configurations involving oxygen, where 80 Ry was used. These cut-off energies were determined after a convergence analysis. The integrations over the Brillouin-zone (BZ) were performed using scheme proposed by Monkhorst-Pack [Monkhorst and Pack(1976)] with a grid of $3 \times 3 \times 1 \mathbf{k}$ points. Norm conserving pseudopotentials generated with fully relativistic calculations including spin-orbit interaction were used after extensive tests of their quality.

A supercell is used to avoid the interaction of the molecules with its periodic images (Figure 1) and it is separated from the adjacent layer by a distance of 45 bohr to prevent interlayer interactions. The optimized lattice constant of monolayer $\mathrm{MoS}_{2}$ is $a=3.24 \AA$. During the structure optimization only the adsorbed molecule and the sulfur atoms near the molecule were allowed to move, in order to save computational effort. The convergence threshold was $10^{-3}$ for all the components of all the forces (in atomic units) plus $10^{-7}$ for the total energy of the system (also in atomic units). We verifyed that these criteria allow a precision of the order of 0.0006 bohr in the relaxed positions, which is enough for our purposes.

The Brillouin-zone integrations involved in calculating the systems' electronic density of states and optical conductivity were performed with a denser $\mathbf{k}$ point grid of $50 \times 50 \times 1 \mathrm{k}$ points and using a gaussian broadening of $0.05 \mathrm{Ry}$.

For the specific case of hydrogen defects in $\mathrm{MoS}_{2}$ we calculated the depth of carrier traps, namely donor and acceptor transitions, by adding or removing electrons to the defective supercell. This was done concurrently with the inclusion of a compensating 

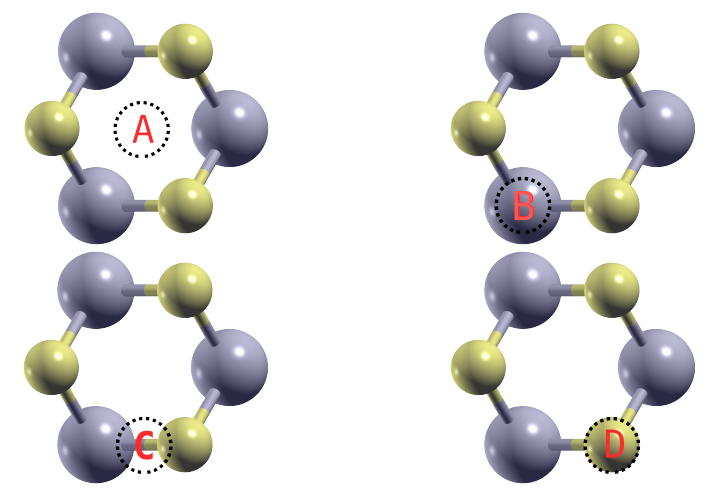

Figure 2. (Color online) Initial sites for the molecules on the monolayer $\mathrm{MoS}_{2}$. At site $\mathrm{A}$ the adsorbant is on the center of the hexagon, at site $\mathrm{B}$ on top of the Mo, at site $\mathrm{C}$ on top of the bound between Mo and $\mathrm{S}$ and at site D on top of $\mathrm{S}$.

uniform charge density across the supercell, so that the integrated charge per cell was effectively zero. Although this is a standard approach, total energies are affected by spurious Coulomb interactions between the artificial array of charged defects. We mitigated these effects by correcting the total energy of charged cells using the method proposed by Freysoldt, Neugebauer, and Van de Walle,[Freysoldt and et al.(2009)] which was recently generalized for anisotropic materials.[Kumagai and Oba(2014)] Accordingly, the Coulomb interaction between charged defects within the $\mathrm{MoS}_{2}$ layer and across vacuum-separated layers was considered to be screened by dielectric constants $\epsilon_{\|}=4.8$ and $\epsilon_{\perp}=1$, respectively. The former value was previously obtained theoretically.[Kumar and Ahluwalia(2012)]

\section{Results and Discussion}

\subsection{Configurations of adsorbants on $M o S_{2}$ monolayer}

To determine the most favorable configurations of the adsorbants on the $\mathrm{MoS}_{2}$ monolayer we placed them on different initial sites of the supercell, and three (four in the case of water and $\mathrm{OH}$ ) different initial orientations. The chosen sites can be seen in figure 2 and are labeled A, B, C and D. For each configuration we let the system relax, so it reconstructs until it reaches an energy minimum. We obtained several metastable configurations for the adsorbants. Table 1 shows the energetics and orientations of the adsorbants in a schematic way in the four positions considered. The adsorption energy was calculated by subtracting to the total energy of the system the sum of the energy of the substrate without adsorbant and the energy of the isolated molecule. Then, for the lowest energy final configuration, we give more details in Table 2, like distances and angles between the adsorbants and the atoms in $\mathrm{MoS}_{2}$. 
Table 1. Adsorption energies $\left(E_{a d s}\right)$ and orientations for the adsorbants in the four reference positions. The first column shows the positions already shown in Figure 2. The third column shows the binding energies for the adsorbants of the second column at the site given in the first. The last column shows the orientation of the axis of the adsorbant: $\mathrm{x}, \mathrm{y}$, and $\mathrm{z}$ are the directions shown in Figure 1; - $\mathrm{z}$ for the $\mathrm{OH}$ means $\mathrm{H}$ is closer to the $\mathrm{MoS}_{2}$ surface in a $\mathrm{z}$ oriented radical; $\mathrm{xz}$ means a line about $45^{\circ}$ between the $\mathrm{x}$ and the $\mathrm{z}$ axis and similar definitions for $\mathrm{xy}$ and $\mathrm{yz}$. Concerning the water molecule, the oxygen atom i allways further away from the $\mathrm{MoS}_{2}$ surface than the hydrogen atoms; $\|$ y means the hydrogen atoms are aligned parallel to the $y$ axis, while the plane of the molecule makes an angle with the normal to the substrate; $\|$ yz means the same hydrogen atoms alignment but the plane of the molecule is parallel to the $y z$ plane; in bold are the energies for the most stable positions, also shown in Table 2. $\mathrm{H}_{2}$ and $\mathrm{OH}$ have no stable position in $\mathrm{C}$.

\begin{tabular}{|c|c|c|c|}
\hline Position & Adsorbant & $\mathrm{E}_{\mathrm{ads}}(\mathrm{eV})$ & Orientation \\
\hline \multirow{4}{*}{$\mathrm{A}$} & $\mathrm{H}_{2}$ & -0.520 & $\mathrm{z}$ \\
\hline & $\mathrm{O}_{2}$ & -0.778 & $\mathrm{xz}$ \\
\hline & $\mathrm{OH}$ & -1.045 & $-\mathrm{Z}$ \\
\hline & $\mathrm{H}_{2} \mathrm{O}$ & -0.795 & $\| \mathrm{y}$ \\
\hline \multirow{4}{*}{ B } & $\mathrm{H}_{2}$ & -0.523 & $\mathrm{z}$ \\
\hline & $\mathrm{O}_{2}$ & -0.784 & $\mathrm{y}$ \\
\hline & $\mathrm{OH}$ & -1.275 & $\mathrm{Z}$ \\
\hline & $\mathrm{H}_{2} \mathrm{O}$ & -0.794 & $\| \mathrm{y}$ \\
\hline \multirow{4}{*}{$\mathrm{C}$} & $\mathrm{H}_{2}$ & - & - \\
\hline & $\mathrm{O}_{2}$ & -0.771 & $\mathrm{xz}$ \\
\hline & $\mathrm{OH}$ & - & - \\
\hline & $\mathrm{H}_{2} \mathrm{O}$ & -0.777 & $\| \mathrm{yz}$ \\
\hline \multirow{4}{*}{$\mathrm{D}$} & $\mathrm{H}_{2}$ & -0.514 & $\mathrm{z}$ \\
\hline & $\mathrm{O}_{2}$ & -0.756 & $\mathrm{xz}$ \\
\hline & $\mathrm{OH}$ & -1.874 & $\mathrm{xz}$ \\
\hline & $\mathrm{H}_{2} \mathrm{O}$ & -0.794 & $\| \mathrm{yz}$ \\
\hline
\end{tabular}

The configurations with the lowest adsorption energy corresponding to the most favorable configurations for each adsorbant are shown in Figure 3.

We first consider the physisorbed molecules, $\mathrm{H}_{2}, \mathrm{O}_{2}$ and $\mathrm{H}_{2} \mathrm{O}$, which have in common being bounded to the $\mathrm{MoS}_{2}$ monolayer by distances larger than 4.5 Bohr. $\mathrm{H}_{2}$ showed very small differences in energies (less than $k_{B} T \simeq 0.0256$ ) for the different sites and orientations. The lowest energy site found had $\mathrm{H}_{2}$ on top of Mo (site B) with an orientation perpendicular to the $\mathrm{MoS}_{2}$ surface, as shown in Figure 3(a). All other sites were also found to be stable except $\mathrm{C}$.

The $\mathrm{O}_{2}$ molecule showed a clear preference to be positioned on top of Mo with an orientation parallel to the $\mathrm{MoS}_{2}$ surface as shown in Figure 3(b). An orientation perpendicular to the surface is unfavorable, but the difference in energy for different 
Table 2. Adsorption energies $\left(\mathrm{E}_{\mathrm{ads}}\right)$ and fully relaxed distances for the adsorbants on the top of the $\mathrm{MoS}_{2}$ monolayer, for the lowest energy configuration. For the meaning of distances and angles, refer to figures 3 and 4 . For the site, refer to figure 2 .

\begin{tabular}{lccccccc} 
Molecule & Site & $\begin{array}{c}\mathrm{E}_{\text {ads }} \\
(\mathrm{eV})\end{array}$ & $\begin{array}{c}d_{1} \\
(\text { bohr })\end{array}$ & $\begin{array}{c}d_{2} \\
(\text { bohr })\end{array}$ & $\begin{array}{c}d_{3} \\
(\text { bohr })\end{array}$ & $\begin{array}{c}d_{4} \\
(\text { bohr })\end{array}$ & $\alpha$ \\
\hline $\mathrm{H}_{2}$ & $\mathrm{~B}$ & -0.52 & 5.04 & 8.04 & 1.42 & & \\
$\mathrm{O}_{2}$ & $\mathrm{~B}$ & -0.78 & 5.61 & 8.61 & 2.33 & & \\
$\mathrm{H}_{2} \mathrm{O}$ & $\mathrm{A}$ & -0.79 & 4.72 & 7.72 & 1.84 & & $48.1^{\circ}$ \\
$\mathrm{HO}$ & $\mathrm{D}$ & -1.87 & 3.14 & 6.14 & 1.86 & 3.27 & $22.0^{\circ}$ \\
$\mathrm{H}$ & $\mathrm{A}$ & -2.60 & 3.03 & 3.62 & & &
\end{tabular}

Table 3. Relative energies of atomic hydrogen in monolayer $\mathrm{MoS}_{2}$, for different charge states $(\mathrm{eV})$. For the configuration labelling, refer to figure 4. For the site, refer to figure 2 .

\begin{tabular}{lccc} 
configuration & - & 0 & + \\
\hline $\mathrm{C}$ & 0.35 & 0.08 & 0.71 \\
$\mathrm{~A}$ & 0.37 & 0.00 & 0.00 \\
$\mathrm{~A}^{\prime}$ & 0.00 & 0.14 & relaxes to A
\end{tabular}

orientations parallel to the surface is smaller than $10 \mathrm{meV}$.

The $\mathrm{H}_{2} \mathrm{O}$ molecule in contrast shows preference for the center of the hexagon (site A). But, contrary to the previous molecules, there are configurations with almost the same energy (a difference smaller than $1 \mathrm{meV}$ ) on sites B and D, although with different orientations. Its most favorable orientation is not totally parallel to $\mathrm{MoS}_{2}$ surface, but making an angle of $48^{\circ}$, as can be seen in Figure 3(d). A position where the oxygen is closer to the surface than the hydrogen atoms is never energetically favorable.

Another possibility is that water is chemisorbed, rather than physisorbed on the surface, dissociating into a $\mathrm{OH}$ radical and a $\mathrm{H}$ atom. The preferred orientation of the $\mathrm{OH}$ radical is shown on Figure 3(c); it forms a bond to the sulfur atom through the oxygen, no matter the original position, and the $\mathrm{H}$ atom is oriented towards the center of the hexagon. Therefore, the $\mathrm{OH}$ group has much lower mobility on the $\mathrm{MoS}_{2}$ monolayer than physisorbed species, since the $\mathrm{OH}$ radical effectively binds to the sulfur atom, forming a chemical covalent bond with the $\mathrm{MoS}_{2}$ monolayer.

The hydrogen atom or proton occupies preferably the A position of the lattice. We considered two other alternative locations, shown in Figure 4: a position near the bond center $(\mathrm{C})$, and a position on the top of $\mathrm{A}$, but displaced outwards from the layer (A'). Even though both of these competing positions are stable in the neutral charge state, they are respectively 0.08 and $0.14 \mathrm{eV}$ higher in energy than the A position for the neutral charge state (Table 3). This is also the most stable position in the negative charge state. In the positive charge state, however, $\mathrm{A}^{\prime}$ is more stable, as hydrogen prefers to sit closer to the more electronegative $\mathrm{S}$ atoms. 


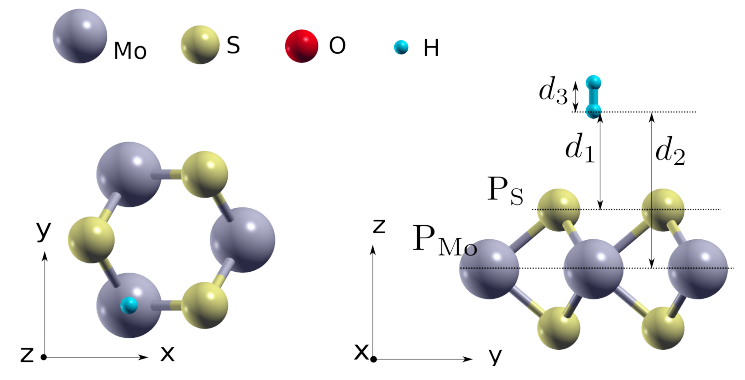

(a) $\mathrm{H}_{2}$
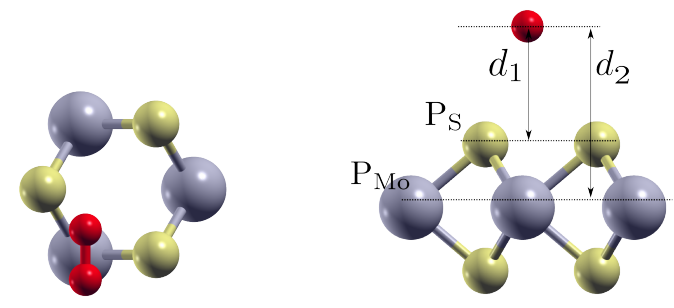

(b) $\mathrm{O}_{2}$
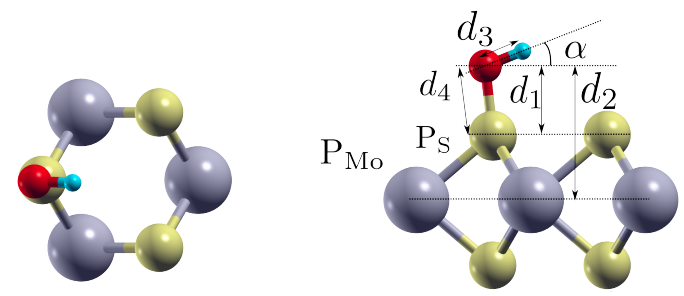

(c) $\mathrm{HO}$
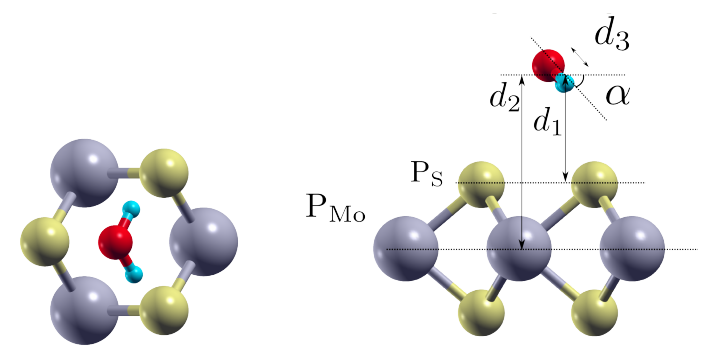

(d) $\mathrm{H}_{2} \mathrm{O}$

Figure 3. (Color online) Monolayer $\mathrm{MoS}_{2}$ with adsorbants on the most favorable position. Left: Top view; Right: side view. On the right side, the distances and angles referred on Table 2 are shown. $\mathrm{P}_{\mathrm{S}}$ and $\mathrm{P}_{\mathrm{Mo}}$ are the sulfur and molybdenum planes, respectively.

\subsection{Electronic states}

After obtaining the most stable configuration for each adsorbant, we determined the electronic structure of these systems. The band diagrams were obtained by going 

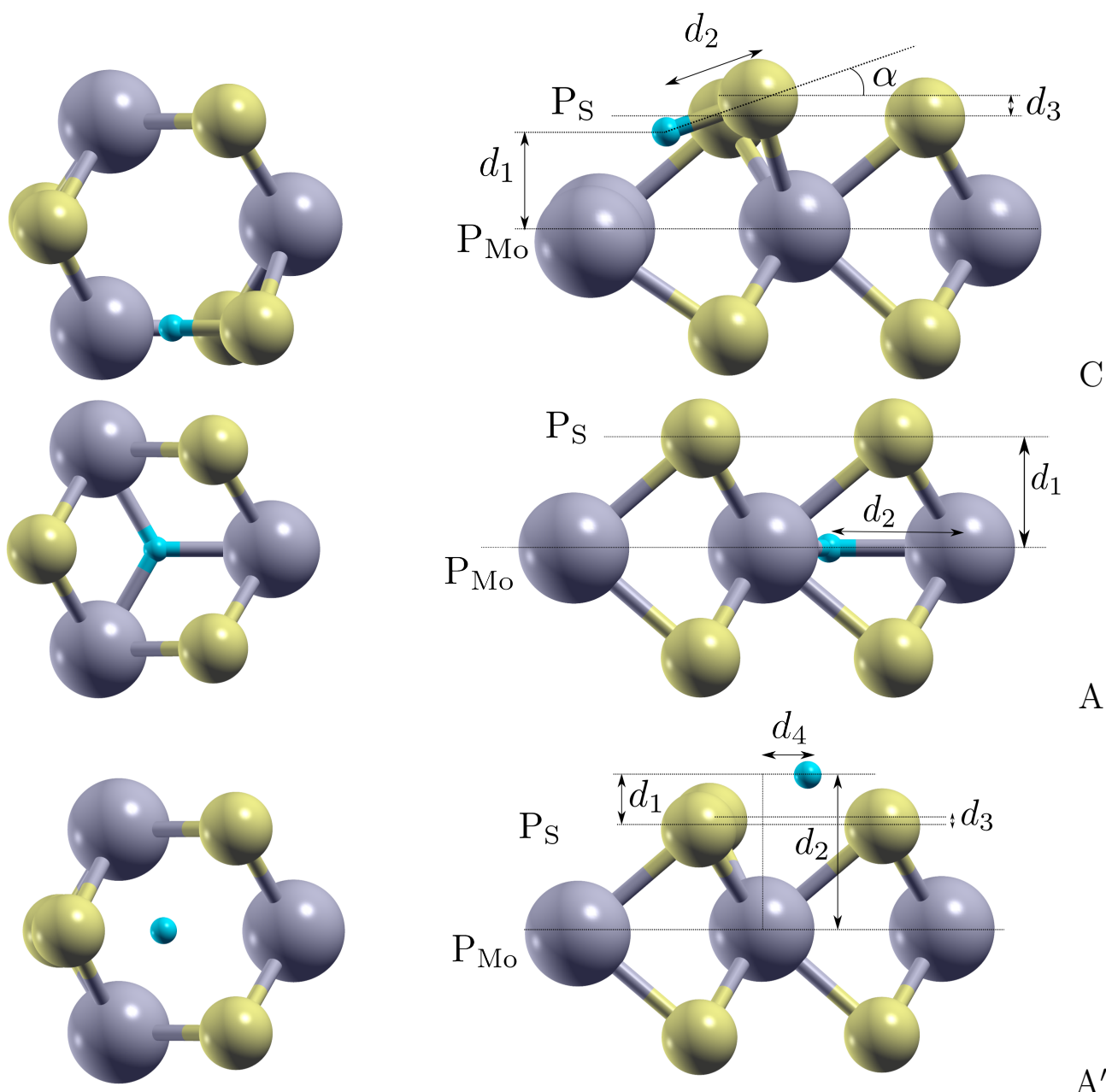

A

$\mathrm{A}^{\prime}$

Figure 4. (Color online) Stable configurations of the hydrogen impurity in monolayer $\mathrm{MoS}_{2}$. The top figure shows configuration C, the middle shows configuration A and the bottom shows configuration $\mathrm{A}^{\prime}$. The distances indicated are given in Table 2 .

from the extreme of the Brillouin Zone (BZ) of the supercell on the y-axis direction of the reciprocal space, to the $\Gamma$ point, and then towards the extreme of the $\mathrm{BZ}$ of the supercell on the $\mathrm{x}$-axis direction of the reciprocal space. This trajectory on the reciprocal space ensures that we pass on the most relevant high symmetry points of the BZ. The particular size of the supercell chosen makes the K-point of the $\mathrm{MoS}_{2}$ monolayer unit cell fold on the top of the $\Gamma$-point (see Figure 5). The top of the valence band is on the K-point (corresponding to the so called A peak of the absorption spectra); the next band is at the $\Gamma$-point, which has an energy between the spin-orbit split bands at the K-point (the blue band of Figure 5); the third band is again at the K-point (corresponding to the so called B peak of the absorption spectra).

Figure 6 (left) shows the band structures of pristine $\mathrm{MoS}_{2}$ monolayer and $\mathrm{MoS}_{2}$ monolayer with adsorbed $\mathrm{H}_{2}$. When adsorbed, the $\mathrm{H}_{2}$ molecule has little influence on the electronic states of the $\mathrm{MoS}_{2}$ monolayer near the gap. It can be seen in Figure 6 (left) that there are no noticeable changes in the valence and conduction bands, and 


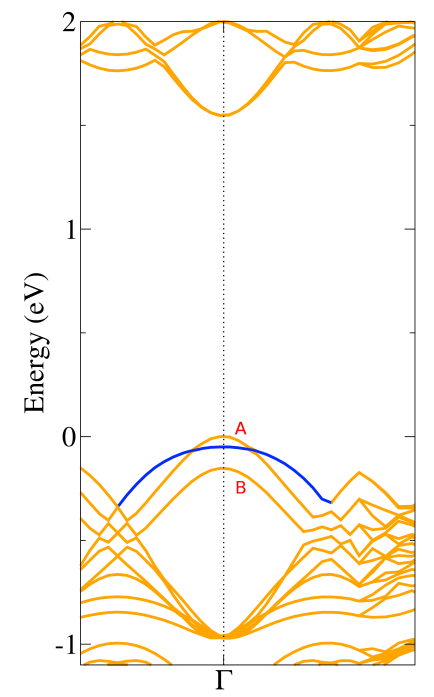

Figure 5. (Color online) Band structure of the $\mathrm{MoS}_{2}$ monolayer supercell. The folding of the bands turns the K-point on the top of the $\Gamma$-point. A and B denote the spin-orbit split bands at the K-point, while the blue band is the band at the $\Gamma$-point.
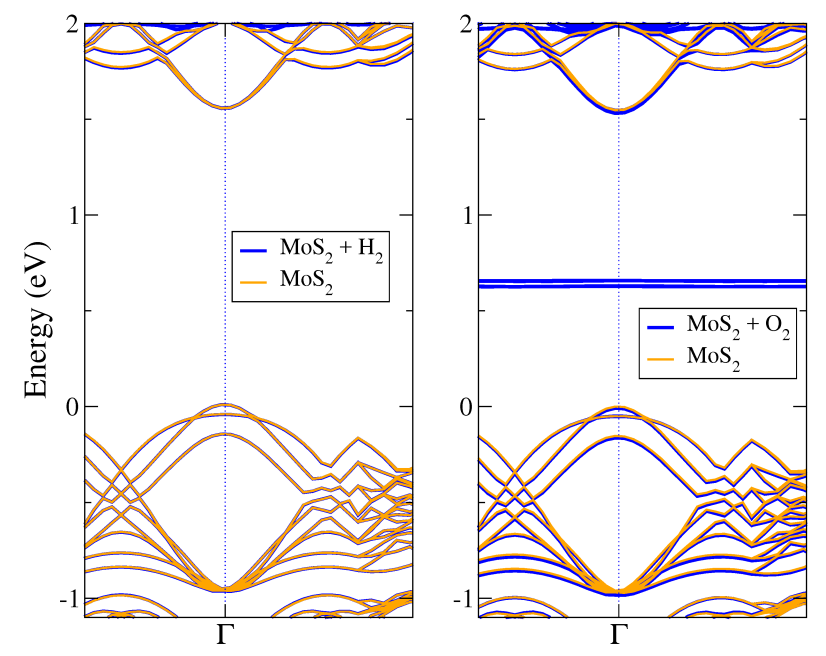

Figure 6. (Color online) Band structure of the pristine $\mathrm{MoS}_{2}$ monolayer (orange) and of $\mathrm{MoS}_{2}$ monolayer with adsorbed $\mathrm{H}_{2}$ (left) and with adsorbed $\mathrm{O}_{2}$ (right). Band structures are overlapped in order to make the differences noticeable.

the gap remains unchanged. The only change we can observe is a deep state with an energy of approximately $-4.5 \mathrm{eV}$, which is too far from the gap to have relevance on $\mathrm{MoS}_{2}$ electronic properties.

In the other hand, the $\mathrm{O}_{2}$ molecule induced sates in the gap, as can be seen in Figure 6 (right). Although it is hard to distinguish in Figure 6, there are four states in the gap, two of them occupied and the other two unoccupied, non-degenerated but with very close energies. The energy difference between the occupied and unoccupied states is $0.03 \mathrm{eV}$. These states may play a role on the absorption spectrum and on scattering of carriers. 

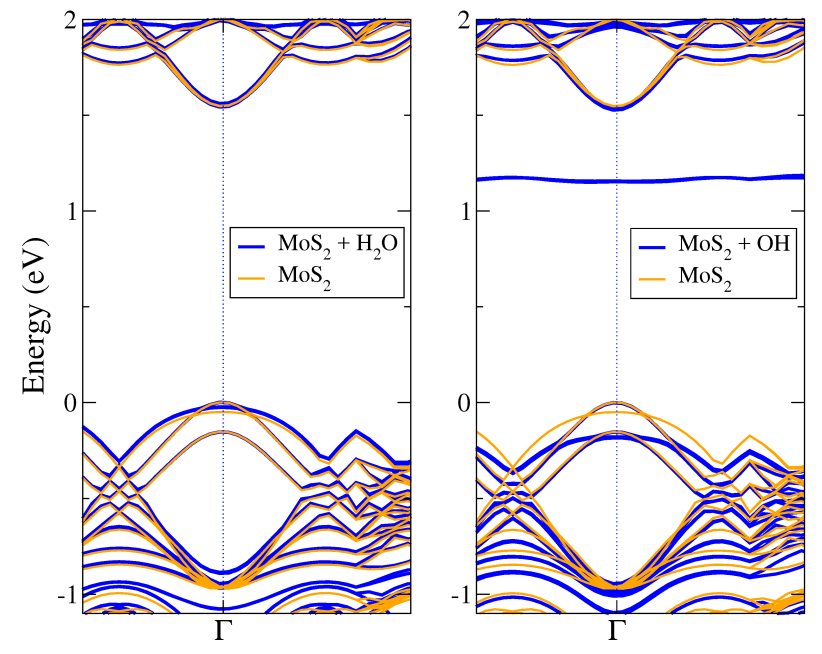

Figure 7. (Color online) Band structure of pristine $\mathrm{MoS}_{2}$ monolayer (orange) and band structure of $\mathrm{MoS}_{2}$ monolayer with adsorbed $\mathrm{OH}$ (left) and with adsorbed $\mathrm{H}_{2} \mathrm{O}$ (right). Band structures are overlapped in order to make the differences noticeable.

The $\mathrm{H}_{2} \mathrm{O}$ molecule, like $\mathrm{H}_{2}$ and $\mathrm{O}_{2}$, did not change much the band structure of the $\mathrm{MoS}_{2}$ monolayer. It can be seen in figure 7 (left) the addition of some states below the valence band. The band at the $\Gamma$-point increases in energy, getting closer to the top of the valence band; however, there was no change in the gap.

In contrast, the $\mathrm{OH}$ and $\mathrm{H}$ radicals clearly alter the electronic properties of the $\mathrm{MoS}_{2}$ monolayer. The bandstructure of $\mathrm{OH}$ on $\mathrm{MoS}_{2}$ is shown in figure 7 (right). Unoccupied defect states are introduced in the gap. Their energies are very close, so we can consider them as quasi-degenerate states. At the $\Gamma$ point, the wave function of the valence band includes a component $p_{z}$ of the atomic wavefunctions of sulfur, which strongly interacts with the $\mathrm{OH}$ radical. The energy of the valence band at the $\Gamma$ point decreased approximately by $0.12 \mathrm{eV}$, and we also observe a small lifting of degeneracy in the same bands. The energy of the quasi degenerate states relative to the valence band is 1.15 $\mathrm{eV}$.

Figure 8 shows the electronic density of states for the systems with adsorbants. The states inside the gap are clearly visible for samples of $\mathrm{MoS}_{2}$ with $\mathrm{OH}$ and $\mathrm{O}_{2}$, as expected. These states contribute significantly to the optical properties of these systems, as can be seen in Fig. 9. Figure 9 shows the calculated optical condutivity. The oxygen molecule contributes with both empty and full states inside the $\mathrm{MoS}_{2}$ gap, leading to transitions both from the valence band to the defect states and from the defects states to the conduction band. This results in the extended absorption band inside the gap that can be seen in Figure 9. The $\mathrm{C}$ peak, also known as the band nesting region,[Carvalho and et al.(2013)] is seen to be considerably suppressed by the adsorbed $\mathrm{O}_{2}$ molecule. The $\mathrm{OH}$ radical also affects the $\mathrm{C}$ peak.

Atomic $\mathrm{H}$ also introduces levels in the gap, and we shall now consider in more detail the charge states of those defects. The ionisation levels, i.e. the value of the Fermi energy for which the defect changes its charge state, is 


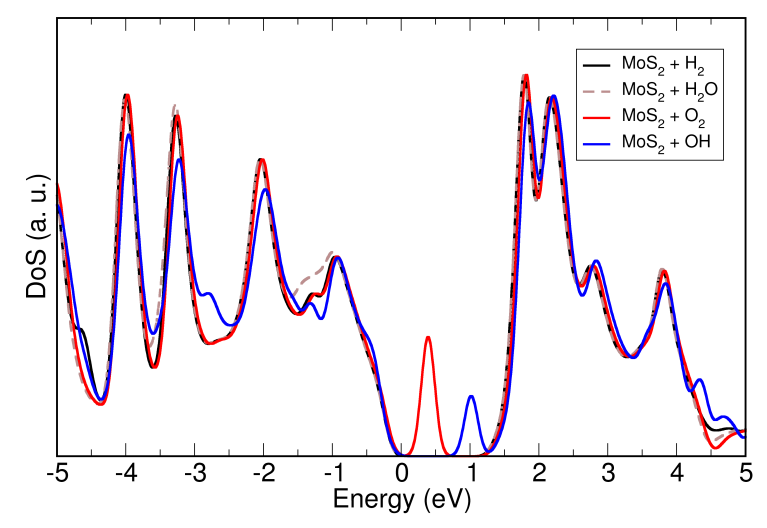

Figure 8. (Color online) Electronic density of states for the systems with adsorbants.

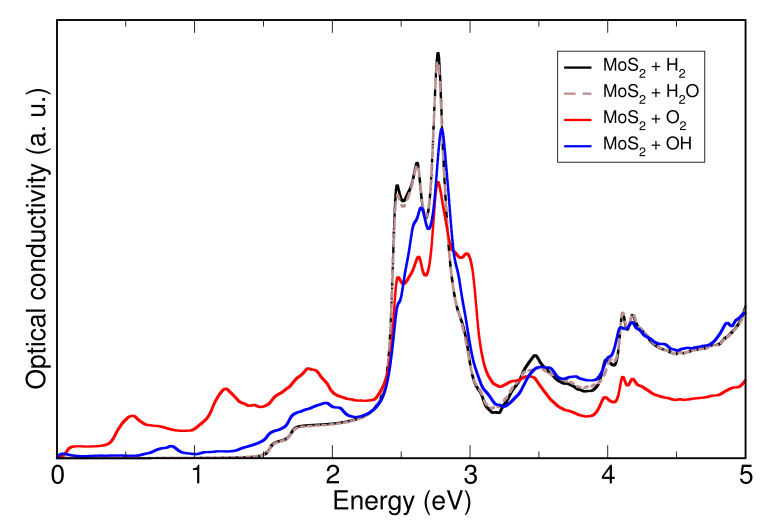

Figure 9. (Color online) Optical conductivity for the systems with adsorbants.

calculated within the marker method,[Coutinho and et al.(2003)] using the perfect supercell as reference. This approach derives from the delta self-consistent $(\triangle \mathrm{SCF})$ method,[Hedin and Lundqvist(1970)] where the valence band top is obtained from the ionization energy of a bulk supercell, $E_{v}=E_{\text {bulk }}(0)-E_{\text {bulk }}(+)$. It can also be demonstrated that when total energies of defective supercells account for periodic charge corrections, the bulk-marker approach is equivalent to the formation energy method as proposed by Qian, Martin, and Chadi.[Qian and et al.(1988)] They differ only on the definition of the valence band top. In the latter method, $E_{v}$ is replaced by the highest occupied Kohn-Sham eigenvalue.

Figure 10 shows how the adsorption energy for atomic hydrogen depends on the Fermi energy $\left(E_{\mathrm{F}}\right)$. On the vertical axis, $E_{\mathrm{F}}$ can vary within a $1.54 \mathrm{eV}$ wide band gap. Gray areas represent valence band and conduction band energies. The vertical solid line at $-2.6 \mathrm{eV}$ stands the adsorption energy of neutral hydrogen at site $\mathrm{A}\left(\mathrm{H}_{\mathrm{A}}^{0}\right)$, whereas lines with negative and positive slope represent the energy of negatively charged and 


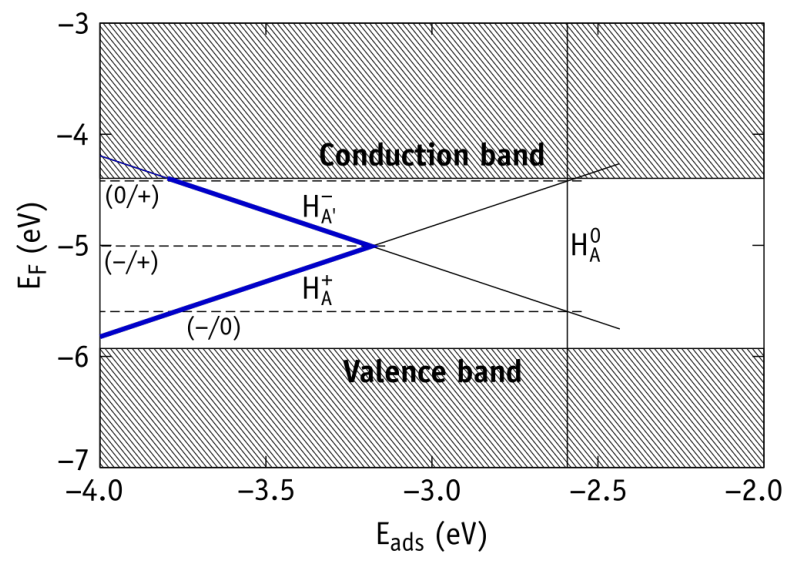

Figure 10. (Color online) Relation between the Fermi level position $\left(E_{\mathrm{F}}\right)$ and the adsorption energy $\left(E_{\text {ads }}\right)$ of atomic hydrogen in monolayer $\mathrm{MoS}_{2}$ (solid lines). Thick and thin lines represent ground state and metastable (excited) states. The dashed line serves as guidance to the location of the $(0 /+),(-/ 0)$ and $(-/+)$ transitions. See text for further details.

positively charged $\mathrm{H}$ at A' and A sites, respectively.

From Figure 10 it becomes clear that hydrogen is an amphoteric impurity - in both n-and p-type materials it may trap majority carriers to become negatively and positively charged, leading to a compensation effect. It is interesting to note that neutral $\mathrm{H}$ is metastable irrespectively of the $E_{\mathrm{F}}$ location. The defect can only be found as a proton at site $\mathrm{A}\left(\mathrm{H}_{\mathrm{A}}^{+}\right)$or as a hydride atom at the $\mathrm{A}^{\prime}$ site $\left(\mathrm{H}_{\mathrm{A}^{\prime}}^{-}\right)$. This is emphasized in Figure 10 as thick blue lines, intersecting at the $(-/+)$ transition calculated at $E_{v}+0.92 \mathrm{eV}$. Hydrogen at site $\mathrm{A}$ is a shallow donor with a level edging the conduction band minimum. This explains the small periodic charge correction of only $E_{\text {corr }}=-0.04 \mathrm{eV}$ for this defect. On the other hand, $\mathrm{H}_{\mathrm{A}^{\prime}}^{-}$has a localized acceptor state, and therefore has a considerably larger charge correction $\left(E_{\text {corr }}=-0.88 \mathrm{eV}\right)$.

Our calculations place the donor and acceptor levels of hydrogen at $E_{v}+1.51 \mathrm{eV}$ and $E_{v}+0.32 \mathrm{eV}$ (see upper and lower dashed lines in Figure 10). These levels are metastable and show a negative- $U$ ordering, like atomic $\mathrm{H}$ does in many other materials.[Van de Walle and Neugebauer(2003)] Thus, hydrogen is unlikely to be the main source of $n$-type conductivity often reported in samples not intentionally doped. Our calculations show that as the Fermi energy raises in the gap, $\mathrm{H}^{-}$will form and compensate any existing shallow donors, including $\mathrm{H}$ at site $\mathrm{A}$.

\section{Conclusions}

The most stable configurations for $\mathrm{H}_{2}, \mathrm{O}_{2}$ and $\mathrm{H}_{2} \mathrm{O}$ molecules on a $\mathrm{MoS}_{2}$ monolayer were determined. It was found that only $\mathrm{O}_{2}$ molecule introduces levels in or close to the bandgap. The most stable configurations for $\mathrm{OH}$ radicals and atomic $\mathrm{H}$ on a $\mathrm{MoS}_{2}$ monolayer were also determined. OH radicals form a chemical covalent bond with the $\mathrm{MoS}_{2}$ monolayer between oxygen atom and sulfur atom, with the hydrogen pointing to 
the center of the hexagon. Atomic $\mathrm{H}$ tends to sit in the middle of the hexagon.

The influence on electronic properties was also studied. $\mathrm{H}_{2}, \mathrm{O}_{2}$ and $\mathrm{H}_{2} \mathrm{O}$ did not change significantly the valence and conduction bands of the $\mathrm{MoS}_{2}$ monolayer. However, the $\mathrm{O}_{2}$ molecule induced two pairs of quasi-degenerate states in the gap zone, one of the pairs being occupied and the other not. The $\mathrm{OH}$ radical changes the valence band at the $\Gamma$ point lowering its energy and also induces empty states in the gap. The relative stability of the charged states of atomic $H$ were determined and it was found that hydrogen is amphoteric with metastable levels at $E(0 /+)=E_{\mathrm{v}}+1.51 \mathrm{eV}$ and $E(-/ 0)=E_{\mathrm{v}}+0.32 \mathrm{eV}$. These show a negative- $U$ ordering, produce a $(-/+)$ thermodynamic transition at $E_{v}+0.92 \mathrm{eV}$, and therefore hydrogen is able to compensate any deliberate doping.

In summary, $\mathrm{O}_{2}$ and $\mathrm{OH}$ influence the electronic and optical properties of the $\mathrm{MoS}_{2}$ monolayer. The fact that they induce states in the gap zone changes the electronic properties of the $\mathrm{MoS}_{2}$ monolayer, and makes this material sensitive to these molecules. Particularly, water dissociation leads to the formation of covalently bonded $\mathrm{OH}$ and interstitial $\mathrm{H}$, both of them electrically active. The control of the $\mathrm{OH}$ and $\mathrm{H}$ charge states via the Fermi level may be used to control the catalisis activity or for the preparation of suitable intermediate steps for further functionalization reactions.

\section{Acknowledgments}

R. M. Ribeiro acknowledge support from the European Commission through the project "Graphene-Driven Revolutions in ICT and Beyond" (Ref. No. 696656), and for the financial support by FEDER through the COMPETE2020 Program, project PTDC/FIS-NAN/3668/2014, and the Portuguese Foundation for Science and Technology (FCT) in the framework of the Strategic Financing UID/FIS/04650/2013 and UID/CTM/50025/2013. Í. J. M. Moura gratefully acknowledges CAPES scholarship process $n^{\circ}$ 99999.001250/2015-09. We gratefully acknowledge that some of the firstprinciples calculations were carried out on the GRC high-performance computer facilities.

\section{References}

[Lee and et al.(2012)] Y.-H. Lee and et al., Adv. Mater. 24, 2320 (2012).

[Kim and et al.(2015)] J. H. Kim and et al., Appl. Phys. Lett. 106, 251606 (2015).

[Kim and et al.(2016)] Y. Kim and et al., J. Phys.: Condens. Matter 28, 184002 (2016).

[Mohapatra and et al.(2016)] P. K. Mohapatra and et al., Appl. Phys. Lett. 108, 042101 (2016).

$[\operatorname{Li}(2015)]$ X. e. a. Li, \bibfield journal \bibinfo journal Nanoscale $\backslash \backslash$ textbf $\backslash$ bibinfo volume $7, \backslash \backslash$ bibinfo pages 8398 ( \bibinfo year 2015).

$[\mathrm{Ma}(2016)]$ D. e. a. Ma, \bibfield journal \bibinfo journal J. Mater. Chem. $\mathrm{C} \backslash \backslash$ textbf $\backslash$ bibinfo volume $4, \backslash \backslash$ bibinfo pages 7093 ( \bibinfo year 2016).

[Chen(2015)] W. e. a. Chen, \bibfield journal \bibinfo journal Journal of the American Chemical

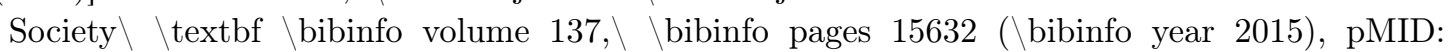
26623946, http://dx.doi.org/10.1021/jacs.5b10519 . 
[Ye(2016)] G. e. a. Ye, \bibfield journal \bibinfo journal Nano Letters \ \textbf \bibinfo volume 16, \ \bibinfo pages 1097 ( \bibinfo year 2016), pMID: 26761422, http://dx.doi.org/10.1021/acs.nanolett.5b04331 .

[Giannozzi and et al.(2009)] P. Giannozzi and et al., \bibfield journal \bibinfo journal Journal of

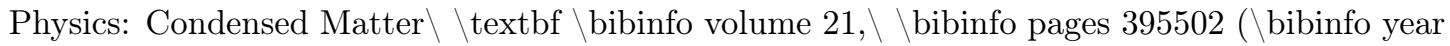
2009).

[Perdew and et al.(1996)] J. P. Perdew and et al., \bibfield journal \bibinfo journal Phys. Rev. Lett. $\backslash$ textbf $\backslash$ bibinfo volume $77, \backslash \backslash$ bibinfo pages 3865 ( \bibinfo year 1996).

[Grimme(2006)] S. Grimme, J. Comput. Chem. 27, 1787 (2006).

[Barone and et al.(2009)] V. Barone and et al., J. Comput. Chem. 30, 934 (2009).

[Monkhorst and Pack(1976)] H. J. Monkhorst and J. D. Pack, \bibfield journal \bibinfo journal Phys.

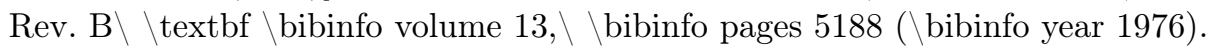

[Freysoldt and et al.(2009)] C. Freysoldt and et al., Phys. Rev. Lett. 102, 016402 (2009).

[Kumagai and Oba(2014)] Y. Kumagai and F. Oba, Phys. Rev. B 89, 195205 (2014).

[Kumar and Ahluwalia(2012)] A. Kumar and P. K. Ahluwalia, Materials Chemistry and Physics 135, 755 (2012).

[Carvalho and et al.(2013)] A. Carvalho and et al., \bibfield journal \bibinfo journal Phys. Rev. B $\backslash$

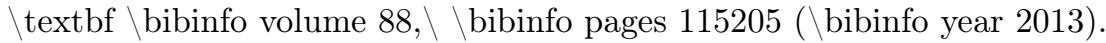

[Coutinho and et al.(2003)] J. Coutinho and et al., \bibfield journal \bibinfo journal Phys. Rev. B $\backslash$

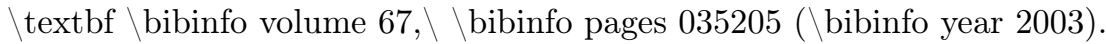

[Hedin and Lundqvist(1970)] L. Hedin and S. Lundqvist, Solid State Physics 23, 1 (1970).

[Qian and et al.(1988)] G.-X. Qian and et al., Physical Review B 38, 7649 (1988).

[Van de Walle and Neugebauer(2003)] C. G. Van de Walle and J. Neugebauer, Nature 423, 626 (2003). 